using siRNA increases VSMC mineralisation (both $\mathrm{P}<0.001$ ). These increases are prevented by inhibiting $\mathrm{TGFI}^{2}$ signalling with SB431542, suggesting cross-talk between FGF-2 and TGFI' ${ }^{2}$ signalling is crucial for the regulation of VSMC mineralisation. Syndecan-4 can also regulate FGF-2 signalling via protein kinase $\mathrm{C} \alpha(\mathrm{PKC} \alpha)$ activation. Biochemical inhibition of PKC $\alpha$ activity using G6976, or knocking-down PKC $\alpha$ expression increases VSMC mineralisation (both $\mathrm{P}<0.05$ ); this increase is also prevented with SB431542. Finally, the ability of FGF-2 to inhibit VSMC mineralisation is reduced when PKC $\alpha$ expression is knocked-down.

In conclusion, our study has identified that syndecan-4/FGF-2 signalling is up-regulated in mineralising VSMCs to reduce $\mathrm{TGF}^{2}$ signalling and minimise further calcification. Syndecan-4 regulates FGF-2 signalling to prevent excessive mineralisation by (a) acting as a co-receptor for FGF-2 and inducing downstream signalling via FGFR and (b) interaction with PKo. The syndecan4/FGF-2/TGF $\alpha$ signalling axis could therefore represent a new therapeutic target for vascular calcification.

\section{CHARACTERISING FUNCTIONAL HETEROGENEITY IN HUMAN EPICARDIUM}

${ }^{1}$ Sophie McManus*, 'Laure Gambardella, ${ }^{1}$ Victoria Moignard, 'Dharini lyer, ${ }^{1}$ Bertie Gottgens, ${ }^{2}$ Nicolas Le Novere, 'Helle Jorgensen, 'Sanjay Sinha. 'University of Cambridge; ${ }^{2}$ Babraham Institute

\subsection{6/heartjnl-2017-311726.209}

Introduction The epicardium is a heterogeneous cell layer covering the mammalian heart. During embryogenesis, the epicardial lineage is essential to heart and vascular development, yielding cardiac fibroblasts and coronary vascular smooth muscle cells. The epicardium also has a trophic effect on developing cardiomyocytes. It is quiescent in adulthood but reactivates post-injury to a limited degree to yield cardiac fibroblasts, which allows fast healing, yet also causes fibrosis. Epicardial functional heterogeneity remains incompletely characterised.

Methods The Sinha group has derived a robust model of human epicardium (hpsc-epi) from human pluripotent stem cells; this was used for single-cell RNA sequencing (scRNAseq). Immunohistochemistry and immunocytochemistry were used to validate scRNA-seq results in primary human foetal tissue. The candidate gene BNC1 has been investigated by siRNA-mediated knockdown studies in vitro.

Results Single-cell RNA-seq identified two main epicardial subpopulations in hpsc-epi: WT $1^{\text {high }} / \mathrm{BNC} 1^{\text {high }} / \mathrm{TCF} 21^{\text {low }}$ and WT $1^{\text {low }} / \mathrm{BNC} 1^{\text {low }} / \mathrm{TCF} 21^{\text {high }}$. Here we show validation of our scRNA-seq data in human foetal epicardium by immunohistochemistry in cryosections and human foetal epicardial explants, confirming our hpsc-epi model is representative of the in vivo situation. We show preliminary data from siRNA-mediated knockdown of BNC1, which indicate this gene may play a role in epicardial function, possibly in regulating cell migration in a model of epithelial-to-mesenchymal transition.

Implications Improved understanding of developmental epicardial regulation could pave the way towards harnessing epicardial potential in prospective strategies to aid revascularisation and regeneration of the injured heart.

\section{SUPPRESSOR OF CYTOKINE SIGNALLING 3 (SOCS3) INTERACTION WITH CAVIN-1 LINKS SOCS3 FUNCTION AND CAVIN-1 STABILITY}

${ }^{1}$ Jamie Williams*, ${ }^{2}$ Nasser Alotaiq, ${ }^{1}$ Tim Palmer. ${ }^{1}$ School of Pharmacy, University of Bradford; ${ }^{2}$ Institute of Cardiovascular and Medical Sciences, University of Glasgow

\subsection{6/heartjnl-2017-311726.210}

Caveolae are lipid raft microdomains essential for the compartmentalisation and regulation of several signalling pathways e.g. JAK/STAT signalling. Disruption of caveolae is a significant factor in multiple disorders including muscular dystrophy, cardiovascular disease, and cancer. Central to caveolae stability is cavin-1 which couples caveolae to the microtubule network to prevent degradation of a key structural element i.e. caveolin-1, and caveolae disassembly. Via an unbiased quantitative proteomics screen, we have identified SOCS3, a negative regulator of JAK/STAT signalling, as a novel cavin-1 interactor.

SOCS3-cavin-1 interactions were characterised by immunoprecipitation assays and probing overlapping peptide arrays. SOCS3 bound to multiple regions within cavin-1, while a PEST motif within the C-terminal region of the SOCS3 $\mathrm{SH} 2$ domain was required for interaction with cavin-1 independently of its capacity to bind phospho-tyrosine. Biochemical analysis and confocal imaging also demonstrated that SOCS3 localisation within lipid raft microdomains and at the plasma membrane required cavin-1. Interestingly, SOCS3 does not ubiquitinate cavin-1 but instead supports cavin-1/caveolae stability. Moreover, genetic deletion of cavin-1 results in the loss of SOCS3-mediatied inhibition of cytokine signalling. Importantly, while the inhibitory function of SOCS3 relies on its induction, caveolae stabilisation occurs at basal SOCS3 expression levels. Thus, transmission electron microscopy demonstrated that SOCS3 knock-out endothelial cells show reduced levels of caveolae.

Our data suggest a novel role for SOCS3 in regulating caveolae assembly while cavin-1, acting as a scaffold-protein, might aid SOCS3-dependent regulation of JAK/STAT signalling. This is the first indication of a novel role for SOCS3 in caveola homeostasis and suggests that loss of caveolae represents a novel mechanism by which chronic activation of pro-inflammatory JAK/STAT signalling could be triggered in disease.

\section{INCREASED BETA-AMYLOID PRODUCTION IS ASSOCIATED WITH DIABETES-INDUCED VASCULAR DYSFUNCTION}

Paul Meakin*, Zofia Tuharska, Christopher McCaffery, Faisel Khan, Michael Ashford. University of Dundee

\subsection{6/heartjnl-2017-311726.211}

Introduction $\beta$-amyloid $(A \beta)$ is produced via the cleavage of amyloid precursor protein $\beta$-secretase (BACE1), resulting in the formation of amyloid plaques, a hallmark pathology of Alzheimer's disease (AD). AD, type 2 diabetes, obesity and cardiovascular disease appear intimately linked with endothelial dysfunction, inflammation, insulin resistance and elevated $\mathrm{A} \tilde{\mathrm{A}} f \AA$, levels all common features. Impaired endothelial function is represented by impaired endothelium-dependent, nitricoxide (NO)-mediated relaxation. Tight regulation of vasoactive 
compounds such as endothelin-1 (ET-1) and NO are required to maintain vascular tine. An imbalance, resulting in increased ET-1 and reduced NO levels promotes vascular stiffening and consequently diseases such as diabetic retinopathy, nephropathy and atherosclerosis. Diseases caused by an increased atherosclerosis burden including coronary heart disease and stroke are major causes of death in obese and diabetic populations.

Aims Determine (1) whether enhanced $A \beta$ levels are sufficient to induce vascular dysfunction and (2) if reducing $A \beta$ production can reverse diet-induced vascular dysfunction.

Methods Measurements of vascular function was determined in vivo by the vascular response to Acetylcholine (endothelial dependent or Sodium Nitroprusside (endothelial independent) using laser Doppler imaging in two studies; (i) Wild-type $(\mathrm{C} 57 \mathrm{BL} / 6)$ mice fed a regular chow diet were infused with murine $\alpha \beta 42$ or scrambled peptide $(\mathrm{ScP} ; 3.36 \mu \mathrm{g} / \mathrm{kg}$ ) in aCSF for 6 weeks or (ii) a BACE1 inhibitor $(\mathrm{M}-3 ; 10 \mathrm{mg} / \mathrm{kg})$ or vehicle (DMSO/PBS) into diet-induced obese (DIO) C57BL/6 mice. Western blotting and ELISAs were used to measure vascular NO signalling and $\mathrm{A} \beta$ production.

Results Circulating levels of $A \beta 42$, not the more prevalent $A \beta 40$ isoform, are increased in both high fat fed mice and obese/diabetic human patients. Infusion of $\mathrm{M}-3$ into DIO mice rescued endothelial dependent reactivity $(\mathrm{M}-327.1 \pm 5.9$, vehicle $1.3 \pm$ 2.9; $\mathrm{P}<0.01)$. In contrast, infusion of $\mathrm{A} \beta 42$ promoted impaired

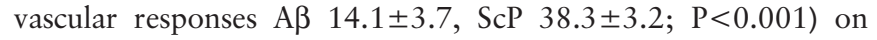
regular chow with no change in body weight. In line with our hypothesis infusion of $\mathrm{A} \beta 42$ increases the ET- $1 / \mathrm{NO}$ ratio $(\mathrm{ScP}$ $1.12 \beta \pm 0.05, \mathrm{~A} \beta 425.35 \pm 0.89 ; \mathrm{P}<0.001$ ), while DIO mice treated with a BACE1 inhibitor, thus with reduced plasma $\mathrm{A} \beta 42$ levels, have a low ET-1/NO ratio (M-3 $1.29 \pm 0.9$, DIO $4.6 \pm$ $1.3 ; \mathrm{P}<0.05)$.

Conclusions We suggest that amyloid processing has a role in normal vascular function with aberrant processing leading to endothelial dysfunction and hypertension. Here we show that pharmacological inhibition of BACE1 can reverse diet-induced endothelial dysfunction, via modulation of plasma $A \beta 42$ levels, as infusion of $\mathrm{A} \beta 42$ can promote the dysfunction independent of a high fat diet.

\section{A HISTONE DEACETYLASE 7-DERIVED 7-AMINO ACID PEPTIDE ACTS AS A PHOSPHORYLATION CARRIER}

Junyao Yang, Ana Moraga*, Yanhua Hu, Lingfang Zeng. King's College London

\subsection{6/heartjnl-2017-311726.212}

Histone deacetylase 7 (HDAC7) belongs to the class II HDAC family and plays a pivotal role in the maintenance of endothelium integrity. There are 8 splicing variants in mouse HDAC7 mRNAs. Within the 5' terminal non-coding area of some variants, there exist some short open reading frames (sORFs). Whether these sORFs can be translated and whether the resulting peptides play roles in cellular physiology remain unclear. In this study, we demonstrated that one sORF encoding a 7-amino-acid (7-aa) peptide could be translated in vascular progenitor cells (VPCs). Importantly, this 7 -aa peptide (7A) could transfer the phosphate group from the phosphorylated Ser393 site of MEKK1 to the Thr145 site of 14-3-3 $\gamma$ protein. The phosphorylated 7A (7Ap) could then directly phosphorylate $14-3-3 \gamma$ protein in a cell-free, in-gel buffer system. The adjacent histidine and proline residues are essential for the phosphate group reception and transfer. In vitro functional analyses revealed that 7A and 7Ap increased VPC self-renewal and migration and enhanced vascular endothelial growth factor (VEGF)-induced VPC migration and differentiation toward the endothelial cell (EC) lineage, in which MEKK1 and 14-3-3 $\gamma$ served as the upstream kinase and downstream effector, respectively. Knockdown of either MEKK1 or 14-3-3 $\gamma$ attenuated VEGF-induced VPC migration and differentiation. Exogenous 7Ap could rescue the effect of VEGF on the MEKK1 siRNA-transfected VPCs but not on the 14-3-3 $\gamma$ siRNA-transfected VPCs. In vivo studies revealed that 7A, especially $7 \mathrm{Ap}$, induced capillary vessel formation in Matrigel plug assays, increased re endothelialization and suppressed neointima formation in the femoral artery injury model, and promoted foot blood perfusion recovery in the hindlimb ischemia model by increasing Sca1+ cell niche formation. These results indicate that the sORFs within the non-coding area can be translated and that 7A may play an important role in cellular processes, such as proliferation, migration and differentiation, by acting as a phosphorylation carrier.

\section{CARDIAC MACROPHAGE INFILTRATION DURING CHRONIC KIDNEY DISEASE ACCELERATES CARDIOVASCULAR DISEASE}

${ }^{1}$ Ana Isabel Garcia Diaz*, ${ }^{2}$ Eleni Vloumidi, ${ }^{2}$ Alex Sardini, ' Charles Pusey, 'James Tomlinson, ${ }^{1}$ Kevin Woollard. ${ }^{1}$ Imperial College London; ${ }^{2}$ MRC London Institute of Medical Sciences

\subsection{6/heartjn|-2017-311726.213}

The relationship between chronic kidney disease (CKD) and increasing rates of cardiovascular disease and mortality is complex, but is important due to the massive increased risk of cardiovascular events noted in CKD patients. Macrophages have critical roles in kidney and cardiovascular disease. However, since a deeper understanding of macrophage ontogeny and heterogeneity, we wanted to revisit the role of cardiovascular macrophages during CKD.

Using the folate induced nephropathy and 5/6 nephrectomy mouse models of CKD, we have new exciting data showing that during CKD, independent of atherosclerosis, inflammatory macrophages are infiltrating cardiac tissue. Using flow cytometry, RNA profiling, histology and ultrasound, we analysed the phenotype and function of the heart and immune cell infiltrate during CKD.

After 12 weeks of $\mathrm{CKD}, \mathrm{CD} 11 \mathrm{~b}^{\text {pos }} \mathrm{F} 480^{\text {pos }} \mathrm{CD} 169^{\text {neg }}$ monocyte derived macrophages infiltrate heart tissue in large numbers. This is only evident in cardiovascular tissue, with no systemic infiltrate in lungs, spleen, kidney or liver. Interestingly, we also noted an increase in cardiac CD19 ${ }^{\text {pos }}$ B-cell and Gr1 ${ }^{\text {pos }}$ neutrophil infiltrate over the course of CKD. The cellular infiltrate was associated with an increase in cardiac fibrosis markers and decrease in heart function, as shown by decreased ejection fraction. Measuring specific chemokine expression in heart and plasma identified a unique chemokine axis, which may be regulating macrophage and other immune cell recruitment to heart tissue during CKD. Moreover, we confirmed the ontogeny of these cardiac macrophages through Ly6C ${ }^{\text {high }}$ monocyte lineages using CCR2 deficient mice, which improved cardiac function.

This work uncovers a unique pathway that mediates inflammatory monocyte derived macrophage infiltration in the heart during CKD. More work is now being performed to confirm mechanisms and cardiovascular phenotype in our CKD 